\title{
Improved Survival in Medically Treated Chronic Thromboembolic Pulmonary Hypertension
}

\author{
Rintaro Nishimura, MD; Nobuhiro Tanabe, $\mathrm{PhD}$; Toshihiko Sugiura, $\mathrm{PhD}$; \\ Ayako Shigeta, PhD; Takayuki Jujo, MD; Ayumi Sekine, MD; \\ Seiichiro Sakao, PhD; Yasunori Kasahara, PhD; Koichiro Tatsumi, PhD
}

\begin{abstract}
Background: The surgical indication for chronic thromboembolic pulmonary hypertension (CTEPH) has been modified due to recognition of peripheral type CTEPH and changes in surgical methods and skill. Bosentan and sildenafil are used as modern oral therapy $(\bmod \mathrm{Tx})$ in patients with inoperable CTEPH, although it remains unknown whether they have positive effects on survival.
\end{abstract}

\begin{abstract}
Methods and Results: A total of 202 patients were diagnosed with CTEPH at Chiba University Hospital between 1986 and 2010, 100 of whom underwent pulmonary endarterectomy. Seven medically treated patients with pulmonary vascular resistance (PVR) $\leq 300 \mathrm{dyn} \cdot \mathrm{s} \cdot \mathrm{cm}^{-5}$ were regarded as having mild disease. Survival rate was stratified by date of diagnosis (group 1, 1986-1998; group 2, 1999-2004; group 3, 2005-2010), and prognostic factors in the remaining 95 medically treated patients were investigated. Group 3 included the most patients treated with mod Tx (group 1, 9.1\%; group 2, 24.2\%; group 3, 65.0\%) and had significantly better survival than either group 1 or 2 (5-year survival: group 1, 54.6\%; group 2, 69.7\%; group 3, 87.3\%). Patients receiving mod Tx had significantly better survival than those not on mod Tx (5-year survival: $88.9 \%$ vs. $60.2 \%$ ). Multivariate analysis showed that mod Tx, lower PVR, and lack of comorbidity were significant predictors of better outcome.
\end{abstract}

Conclusions: Medically treated patients with CTEPH had a better survival rate, and the use of mod Tx contributed to improved survival. (Circ J 2013; 77: 2110-2117)

Key Words: Chronic thromboembolic pulmonary hypertension; Medical treatment; Survival

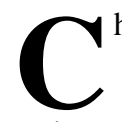
hronic thromboembolic pulmonary hypertension (CTEPH) results from obstruction of pulmonary arteries by unresolved, organized thrombi. Untreated patients with CTEPH may progress to right heart failure and death. A study of patients with inoperable CTEPH showed that prognosis was poor (5-year survival approx imately 30\%), especially in those with a mean pulmonary artery pressure (mPpa) $>40 \mathrm{mmHg} .{ }^{1}$ Pulmonary endarterectomy (PEA) is a promising treatment for surgically accessible thrombi because this condition is potentially curable. ${ }^{2,3}$ Small vessel diseases, however, similar to pulmonary arterial hypertension (PAH) may also exist with CTEPH and contribute to disease progression., ${ }^{4,5}$ Medical therapy for PAH is therefore used in patients with inoperable disease or persistent pulmonary hypertension $(\mathrm{PH})$ after PEA. ${ }^{6,7}$ A randomized trial in patients with inoperable disease or persistent $\mathrm{PH}$ after surgery showed that the endothelin receptor antagonist (ERA), bosentan, had significant positive hemodynamic effects, but did not significantly increase 6-min-walk distance (6MWD) compared with a placebo group. ${ }^{7}$
A recent study by Condliffe et al reported improved survival in a higher proportion of patients treated with ERA and phosphodiesterase-5 inhibitors (PDE-5I). ${ }^{8}$

\section{Editorial p 1990}

The mortality rate associated with PEA has decreased as a consequence of improved surgical skills and patient selection. ${ }^{3,8}$ PEA using a thoracotomy approach was started Chiba University Hospital in 1986, with a median sternotomy used since 1990. Initially all patients without comorbidities underwent surgery. Due to the high operative mortality of patients with high pulmonary vascular resistance (PVR) and the introduction of medical therapy, prior to 2004 patients with PVR $>1,100 \mathrm{dyn} \cdot \mathrm{s} \cdot \mathrm{cm}^{-5}$ were frequently excluded from surgery. Recently, only patients with high PVR without a substantial obstruction are excluded. ${ }^{9}$ Because the indications for surgery have modified over time, the characteristics of patients suitable for medical treatment now differ from those in the past.

Received November 9, 2012; revised manuscript received March 12, 2013; accepted March 24, 2013; released online April 25, 2013 Time for primary review: 24 days

Department of Respirology, Graduate School of Medicine, Chiba University, Chiba, Japan

Grant: See acknowledgments.

Mailing address: Rintaro Nishimura, MD, Department of Respirology, Graduate School of Medicine, Chiba University, 1-8-1 Inohana,

Chuou-ku, Chiba 260-8670, Japan. E-mail: rintaro09281980@yahoo.co.jp

ISSN-1346-9843 doi:10.1253/circj.CJ-12-1391

All rights are reserved to the Japanese Circulation Society. For permissions, please e-mail: cj@j-circ.or.jp 
It has been shown in Western countries that the presence of associated medical conditions (AMC) such as splenectomy, ventriculo-atrial shunt, inflammatory bowel disease, and osteomyelitis are linked to the development of CTEPH and poor survival in both surgically and medically treated patients. ${ }^{10}$ There are no reports, however, on the influence of AMC and comorbidity on survival of CTEPH patients from Asian countries.

We have encountered many patients who were excluded from surgery and were then followed up for $>20$ years on medical treatment. The current study investigated whether the survival rate of medically treated patients has increased and, if so, whether the increase was dependent on the characteristics of the patient including hemodynamics, extent of central thrombi and comorbidities, and use of modern oral therapy (mod Tx) such as bosentan and sildenafil. The study also examined whether mod Tx improved long-term survival.

\section{Methods}

\section{Study Design and Subjects}

This study was a retrospective single-center cohort investigation of 202 patients with CTEPH diagnosed consecutively between January 1986 and December 2010 at Chiba University Hospital. CTEPH was defined as $\mathrm{mPpa}>25 \mathrm{mmHg}$ with normal wedge pressure on right heart catheterization in patients with symptoms such as dyspnea on exertion for $>6$ months. Lung perfusion scans were also required to demonstrate segmental or larger defects concomitant with normal ventilation scans. Chronic thromboembolic findings were confirmed on pulmonary angiography. ${ }^{11}$ Of the 202 patients, 100 underwent PEA, while 102 patients were treated medically because they had been excluded from surgery on the basis of the surgical indications at that time. Although the indications for surgery have been modified by improved surgical skills and changes in surgical methods (ie, thoracotomy to median sternotomy), the major reasons for exclusion at that time were mild disease with PVR $\leq 300 \mathrm{dyn} \cdot \mathrm{s} \cdot \mathrm{cm}^{-5}(\mathrm{n}=7)$, relatively peripheral type $(n=72)$, high PVR $(n=3)$, severe comorbidities $(n=14)$, and age $>70$ years or unwillingness to undergo surgery $(n=6)$. Mild disease was excluded from this study because the fair prognosis is already known. ${ }^{1}$

According to Japanese legislation, informed consent is not required for retrospective collection of data that relate to current practice. The study database, however, was anonymized and complied with the requirements of the Ministry of Health, Labor and Welfare, which is dedicated to privacy, information technology, and civil rights in Japan. The Ethics Committee of Chiba University approved the study protocol (approval number 1248). In addition, the study protocol for investigating human leukocyte antigen (HLA) was approved by the Research Ethics Committee of Chiba University School of Medicine, with written, informed consent being obtained from the patients (approval numbers 84 and 220).

\section{Assessment of Central Thrombi}

Central arteries were defined as vessels proximal to segmental branches and were divided into 4 portions. ${ }^{12}$ The central disease score was calculated as the sum of the abnormal central portions, to a maximum score of 4 . Prior to 1997 , this score was determined on conventional computed tomography (CT) and pulmonary angiography, and from then on by helical CT.

\section{Medical Treatment}

Warfarin was prescribed for all medically treated patients to

\begin{tabular}{|c|c|}
\hline Age (years) & $55.3 \pm 13.7$ \\
\hline Gender (F/M) & $75 / 20$ \\
\hline Duration of symptoms (months) & $29.6 \pm 27.9$ \\
\hline $\mathrm{mPra}(\mathrm{mmHg})$ & $5.1 \pm 4.2$ \\
\hline $\mathrm{mPpa}(\mathrm{mmHg})$ & $43.3 \pm 12.0$ \\
\hline $\mathrm{Cl}\left(\mathrm{L} \cdot \mathrm{min}^{-1} \cdot \mathrm{m}^{-2}\right)$ & $2.60 \pm 0.60$ \\
\hline PVR (dyn $\left.\cdot s \cdot \mathrm{cm}^{-5}\right)$ & $792 \pm 407$ \\
\hline $\mathrm{PaO}_{2}$ (torr) & $58.2 \pm 10.9$ \\
\hline WHO functional class $(1 / 2 / 3 / 4)$ & $2 / 31 / 55 / 6$ \\
\hline Central disease score $(0 / 1 / 2 / 3 / 4)$ & $46 / 31 / 13 / 5 / 0$ \\
\hline Comorbidity & 46.3 \\
\hline Coagulation abnormality & 29.5 \\
\hline HLA B5201+ & 39.8 \\
\hline DVT & 34.7 \\
\hline Mod Tx & 37.9 \\
\hline Bosentan (n) & 10 \\
\hline Sildenafil (n) & 17 \\
\hline Bosentan + Sildenafil $(n)$ & 6 \\
\hline Clinical trial & 3 \\
\hline Epoprostenol (n) & 3 \\
\hline Beraprost & 56.8 \\
\hline Oxygen & 88.4 \\
\hline Diuretics & 52.6 \\
\hline Inferior vena cava filter & 50.5 \\
\hline
\end{tabular}

Data given as mean $\pm \mathrm{SD}, \%$ or $\mathrm{n}$.

$\mathrm{Cl}$, cardiac index; DVT, deep vein thrombosis; mod Tx, modern oral therapy; $\mathrm{mPpa}$, mean pulmonary arterial pressure; mPra, mean right atrial pressure; $\mathrm{PaO}_{2}$, arterial oxygen tension; PVR, pulmonary vascular resistance; WHO, World Health Organization.

obtain a target international normalized ratio of prothrombin time between 1.5 and 3 , with $88.4 \%$ of patients receiving ambulatory oxygen therapy and $52.6 \%$ given diuretics. In Japan, epoprostenol and the oral prostanoid, beraprost, have been available since 1999 , bosentan since 2005 , and sildenafil since 2008. In addition, sildenafil was used at Chiba University Hospital in 2005 in a doctor-oriented clinical trial. In the current study we defined bosentan, sildenafil, and new oral drugs undergoing clinical trial as modern Tx.

\section{Survival Analysis}

Patients were classified into 3 groups according to date of diagnosis (group 1, 1986-1998; group 2, 1999-2004; and group $3,2005-2010)$. To investigate the long-term efficacy of medical treatment, the survival rates and prognostic factors were compared in the 3 groups. To evaluate the benefit of mod Tx on survival, we also compared survival from the time of initiation of mod Tx with survival from date of diagnosis in patients not taking mod Tx. Prognostic factors including baseline hemodynamics at the last catheterization prior to initiation of mod Tx were also examined. In December 2010, follow-up data were obtained from 94 of the 95 patients by either contacting them or their primary physicians. Sixty-one patients were still alive and 33 patients had died. Survival time was calculated from the date of diagnosis on right heart catheterization or date of initiation of mod Tx. The remaining 1 patient was censored at the final visit date by their primary physician. In addition, 2 patients who underwent pulmonary balloon angioplasty during follow-up were censored at the first procedure date, although medical treatment was continued after angio- 


\begin{tabular}{|c|c|c|c|c|}
\hline & Group $1(n=22)$ & Group $2(n=33)$ & Group $3(n=40)$ & P-value \\
\hline Age (years) & $53.5 \pm 12.4$ & $54.8 \pm 14.0$ & $56.7 \pm 14.2$ & NS \\
\hline Gender (F/M) & $16 / 6$ & $29 / 4$ & $30 / 10$ & NS \\
\hline Duration of symptoms (months) & $28.1 \pm 23.6$ & $25.6 \pm 24.4$ & $33.6 \pm 32.5$ & NS \\
\hline $\mathrm{mPpa}(\mathrm{mmHg})$ & $40.0 \pm 12.2$ & $41.8 \pm 11.6$ & $46.3 \pm 11.9$ & NS \\
\hline $\mathrm{Cl}\left(\mathrm{L} \cdot \mathrm{min}^{-1} \cdot \mathrm{m}^{-2}\right)$ & $2.70 \pm 0.67$ & $2.58 \pm 0.60$ & $2.57 \pm 0.57$ & NS \\
\hline PVR (dyn $\left.\cdot s \cdot \mathrm{cm}^{-5}\right)$ & $748 \pm 451$ & $789 \pm 455$ & $818 \pm 346$ & NS \\
\hline $\mathrm{PaO}_{2}$ (torr) & $59.9 \pm 12.0$ & $58.6 \pm 12.3$ & $56.9 \pm 9.1$ & NS \\
\hline WHO functional class $(1 / 2 / 3 / 4)$ & 0/8/10/4 & $1 / 9 / 20 / 2$ & $1 / 14 / 25 / 0$ & NS \\
\hline Central disease score $(\leq 1 / \geq 2)$ & $13 / 9$ & $29 / 4$ & $\overrightarrow{35 / 5}$ & 0.01 \\
\hline Comorbidity & 50.0 & 42.4 & 47.5 & NS \\
\hline HLA B5201+ & 46.7 & 45.2 & 32.4 & NS \\
\hline DVT & 22.7 & 24.2 & $\overrightarrow{50.0}$ & 0.03 \\
\hline Mod Tx & $\begin{array}{c}9.1 \\
\llcorner\end{array}$ & $\begin{array}{r}24.2 \\
\square\end{array}$ & $\stackrel{65.0}{\longrightarrow}$ & $<0.0001$ \\
\hline Bosentan (n) & 0 & 3 & 7 & \\
\hline Sildenafil (n) & 1 & 4 & 12 & \\
\hline Bosentan + Sildenafil $(n)$ & 1 & 0 & 5 & \\
\hline Clinical trial & 0 & 1 & 2 & \\
\hline Epoprostenol (n) & 0 & 3 & 0 & \\
\hline Beraprost & 36.4 & $\stackrel{75.8}{\sqcup}$ & 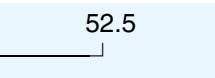 & 0.01 \\
\hline Oxygen & $\begin{array}{c}72.7 \\
\llcorner \\
\end{array}$ & $\stackrel{93.9}{\longleftarrow}$ & 92.5 & 0.03 \\
\hline Diuretics & 66.7 & 57.6 & 47.5 & NS \\
\hline Inferior vena cava filter & 45.5 & 57.6 & 47.5 & NS \\
\hline
\end{tabular}

Data given as mean $\pm S D, \%$ or $n$. ${ }^{*} P<0.01 ;{ }^{\$} P<0.05$. Abbreviations as in Table 1 .

plasty.

\section{Statistical Analysis}

The results are expressed as mean \pm SD for continuous variables and as number and percentage for categorical variables. Comparison of the groups was performed using 1-way factorial ANOVA with Bonferroni correction for multiple comparisons of continuous variables. Chi-squared test was used for comparison of categorical variables. Survival curves were analyzed on the Kaplan-Meier method, and the Wilcoxon test used to compare survival. Univariate and multivariate Cox proportional hazard models were used to examine prognostic factors and the independent effect on survival of date of diagnosis or use of mod Tx. $\mathrm{P}<0.05$ was considered statistically significant. All statistical analysis was performed using commercially available software (JMP 9.0.0, Japanese version, SAS Institute Tokyo, Japan).

\section{Results}

The mean age of the 95 medically treated patients was $55.3 \pm$ 13.7 years, with a greater number of women $(n=75)$ than men $(n=20)$. The interval from onset of symptoms to cardiac catheterization was $29.6 \pm 27.9$ months. mPpa and PVR were 43.3 $12.0 \mathrm{mmHg}$ and $792 \pm 407 \mathrm{dyn} \cdot \mathrm{s} \cdot \mathrm{cm}^{-5}$, respectively (Table 1 ). Comorbidities that may have affected prognosis included thyroid disease $(n=10)$, cancer $(n=8)$, psychiatric diseases $(n=7)$, chronic obstructive pulmonary disease $(n=5)$, coronary artery disease $(n=4)$, ischemic stroke $(n=3)$, non-tuberculosis myco- bacterium infection $(n=4)$, epilepsy $(n=3)$, primary thrombocythemia $(n=3)$, severe diabetes mellitus $(n=2)$, AMC $(n=1)$, and thrombocytopenia $(n=1)$.

As shown in Table 2, a history of deep vein thrombosis (DVT) was more prevalent in group 3 than in groups 1 and 2. Group $1(59.1 \%)$ included fewer patients with the relatively peripheral type CTEPH compared with group $2(87.9 \% ; \mathrm{P}=0.01)$ and group $3(87.5 \% ; \mathrm{P}=0.01)$. Beraprost (average dose $104.0 \pm$ $69.8 \mu \mathrm{g} /$ day, range $360-20 \mu \mathrm{g} /$ day, median $80 \mu \mathrm{g} /$ day) was given more often in group $2(75.8 \%)$ than in group $1(36.4 \% ; \mathrm{P}=0.004)$ and group $3(52.5 \% ; \mathrm{P}=0.04)$, while $\bmod \mathrm{Tx}$ (bosentan: average dose, $141.7 \pm 60.1 \mu \mathrm{g} /$ day, range $250-62.5 \mu \mathrm{g} /$ day, median $125 \mu \mathrm{g} /$ day; sildenafil: average dose $53.4 \pm 11.7 \mu \mathrm{g} /$ day, range $60-30 \mu \mathrm{g} /$ day, median $60 \mu \mathrm{g} /$ day) was used more frequently in group $3(65.0 \%)$ compared with group $1(9.1 \%$; $\mathrm{P}<0.0001)$ and group $2(24.2 \% ; \mathrm{P}=0.0005)$. Three patients were registered in a clinical trial using new oral PAH drugs such as riociguat and selexipag, with 2 of these patients receiving sildenafil after the trial. One patient was treated with epoprostenol only and 2 with epoprostenol and sildenafil. No significant differences were observed in the other baseline characteristics.

The 5-year survival rate from the date of diagnosis in all patients was $71.7 \%$. Thirty-three patients died, with the cause of death being right ventricular failure or sudden death $(n=29)$, hemoptysis $(n=1)$, recurrence $(n=1)$, multiple myeloma $(n=1)$, or cerebral bleeding $(n=1)$. Figure 1 shows the survival curves stratified by date of diagnosis. The 5-year survival rate in groups 1,2 and 3 was $54.6 \%, 69.7 \%$, and $87.3 \%$, respectively. Group 3 had a significantly better survival rate compared with 


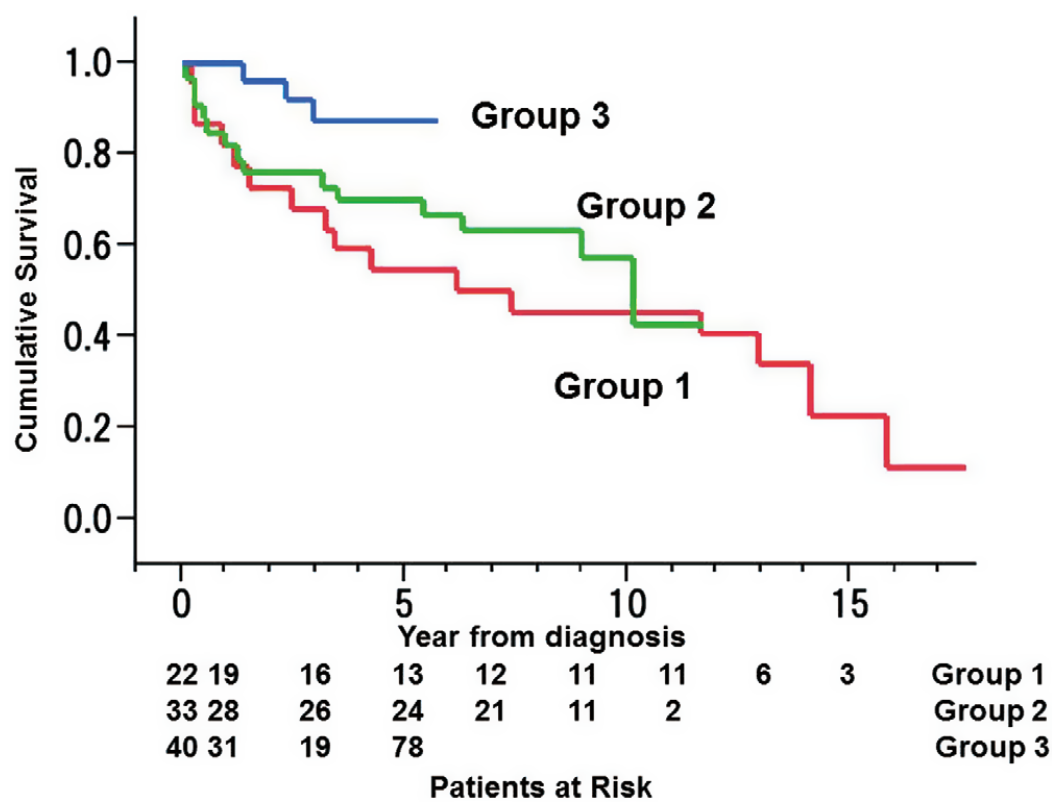

Figure 1. Survival curves according to date of diagnosis $(n=95)$. Group 3 patients (2005-2010) had a significantly better survival rate than either group 1 (1886-1998) or group 2 (1999-2004). The 5 -year survival in group 3 was $87.3 \%$ vs. $54.6 \%$ and $69.7 \%$ in groups 1 and 2 , respectively $(P=0.02$, Wilcoxon test).

\begin{tabular}{|c|c|c|c|c|c|c|c|c|}
\hline \multirow{2}{*}{ Factor } & \multicolumn{2}{|c|}{ Univariate $^{\dagger}$} & \multicolumn{2}{|c|}{ Multivariate $^{\dagger}$} & \multicolumn{2}{|c|}{ Univariate $^{\ddagger}$} & \multicolumn{2}{|c|}{ Multivariate ${ }^{\ddagger}$} \\
\hline & HR (95\% Cl) & P-value & HR (95\% Cl) & P-value & HR (95\% Cl) & P-value & HR $(95 \% \mathrm{Cl})$ & P-value \\
\hline mPpa $(\mathrm{mmHg})$ & $\begin{array}{c}1.02 \\
(0.990-1.045)\end{array}$ & 0.2 & & & $\begin{array}{c}1.02 \\
(0.993-1.054)\end{array}$ & 0.13 & & \\
\hline $\begin{array}{l}\text { Cardiac index } \\
\left(\mathrm{L} \cdot \mathrm{min}^{-1} \cdot \mathrm{m}^{-2}\right)\end{array}$ & $\begin{array}{c}0.61 \\
(0.313-1.136)\end{array}$ & 0.12 & & & $\begin{array}{c}0.55 \\
(0.307-0.992)\end{array}$ & 0.046 & & \\
\hline $\mathrm{PaO}_{2}$ (torr) & $\begin{array}{c}0.96 \\
(0.926-0.996)\end{array}$ & 0.03 & & & $\begin{array}{c}0.97 \\
(0.932-1.001)\end{array}$ & 0.06 & & \\
\hline PVR (dyn $\left.\cdot s \cdot \mathrm{cm}^{-5}\right)$ & $\begin{array}{c}1.001 \\
(1.000-1.002)\end{array}$ & 0.003 & & & $\begin{array}{c}1.001 \\
(1.000-1.002)\end{array}$ & 0.002 & $\begin{array}{c}1.001 \\
(1.001-1.002)\end{array}$ & 0.0007 \\
\hline $\begin{array}{l}\text { PVR }<1,100 \mathrm{dyn} \cdot \mathrm{s} \cdot \mathrm{cm}^{-5} \\
\left(\mathrm{vs} . \geq 1,100 \mathrm{dyn} \cdot \mathrm{s} \cdot \mathrm{cm}^{-5}\right)\end{array}$ & $\begin{array}{c}0.28 \\
(0.136-0.623)\end{array}$ & 0.002 & $\begin{array}{c}0.35 \\
(0.168-0.775)\end{array}$ & 0.01 & & & & \\
\hline CD score 0-1 (vs. 2-4) & $\begin{array}{c}0.31 \\
(0.151-0.651)\end{array}$ & 0.003 & $\begin{array}{c}0.431 \\
(0.136-0.742)\end{array}$ & 0.009 & $\begin{array}{c}0.35 \\
(0.173-0.745)\end{array}$ & 0.008 & $\begin{array}{c}0.54 \\
(0.249-1.203)\end{array}$ & 0.13 \\
\hline $\begin{array}{l}\text { No comorbidity } \\
\text { (vs. comorbidity) }\end{array}$ & $\begin{array}{c}0.33 \\
(0.150-0.680)\end{array}$ & 0.002 & $\begin{array}{c}0.32 \\
(0.143-0.675)\end{array}$ & 0.002 & $\begin{array}{c}0.35 \\
(0.158-0.712)\end{array}$ & 0.004 & $\begin{array}{c}0.39 \\
(0.176-0.809)\end{array}$ & 0.01 \\
\hline $\begin{array}{l}\text { Mod Tx } \\
\text { (vs. without mod Tx) }\end{array}$ & & & & & $\begin{array}{c}0.38 \\
(0.110-0.994)\end{array}$ & 0.049 & $\begin{array}{c}0.31 \\
(0.086-0.887)\end{array}$ & 0.03 \\
\hline \multicolumn{9}{|l|}{ Year at diagnosis } \\
\hline Group 3 (vs. group 1) & $\begin{array}{c}0.21 \\
(0.047-0.677)\end{array}$ & 0.02 & $\begin{array}{c}0.30 \\
(0.059-0.871)\end{array}$ & 0.03 & & & & \\
\hline Group 3 (vs. group 2) & $\begin{array}{c}0.28 \\
(0.065-0.897)\end{array}$ & 0.02 & $\begin{array}{c}0.21 \\
(0.056-0.796)\end{array}$ & 0.03 & & & & \\
\hline
\end{tabular}

†Baseline pulmonary hemodynamics at diagnosis and survival from the date of diagnosis were used; ‡baseline pulmonary hemodynamics at the last catheterization prior to initiation of mod Tx and survival from the time of initiation of mod Tx were used in mod Tx group, while baseline pulmonary hemodynamics at diagnosis and survival from date of diagnosis were used in patients not taking mod Tx. CD score, central disease score; $\mathrm{Cl}$, confidence interval; $\mathrm{HR}$, hazard ratio. Other abbreviations as in Table 1.

groups $1(\mathrm{P}=0.005)$ and $2(\mathrm{P}=0.03)$. The survival curves in groups 1 and 2 were not significantly different.

Univariate Cox proportional hazard analysis showed that diagnosis between 2005 and 2010, low PVR (continuous), PVR $<1,100 \mathrm{dyn} \cdot \mathrm{s} \cdot \mathrm{cm}^{-5}$, central disease score $\leq 1$, and no comorbidity were associated with better outcome. These parameters were also significant predictors of better outcome on multivariate analysis. As shown in Table 3, diagnosis between
2005 and 2010 was an independent predictor of better outcome.

Patients with PVR $\geq 1,100 \mathrm{dyn} \cdot \mathrm{s} \cdot \mathrm{cm}^{-5}$ had a significantly poorer survival than those with PVR $<1,100 \mathrm{dyn} \cdot \mathrm{s} \cdot \mathrm{cm}^{-5}(5-$ year survival rate: $31.6 \%$ vs. $78.0 \%, \mathrm{P}=0.001$; Figure 2 ). This cut-off for PVR was determined previously in a study that found that PVR $\geq 1,100 \mathrm{dyn} \cdot \mathrm{s} \cdot \mathrm{cm}^{-5}$ was associated with high perioperative mortality in CTEPH patients. ${ }^{13}$ Receiver operat- 


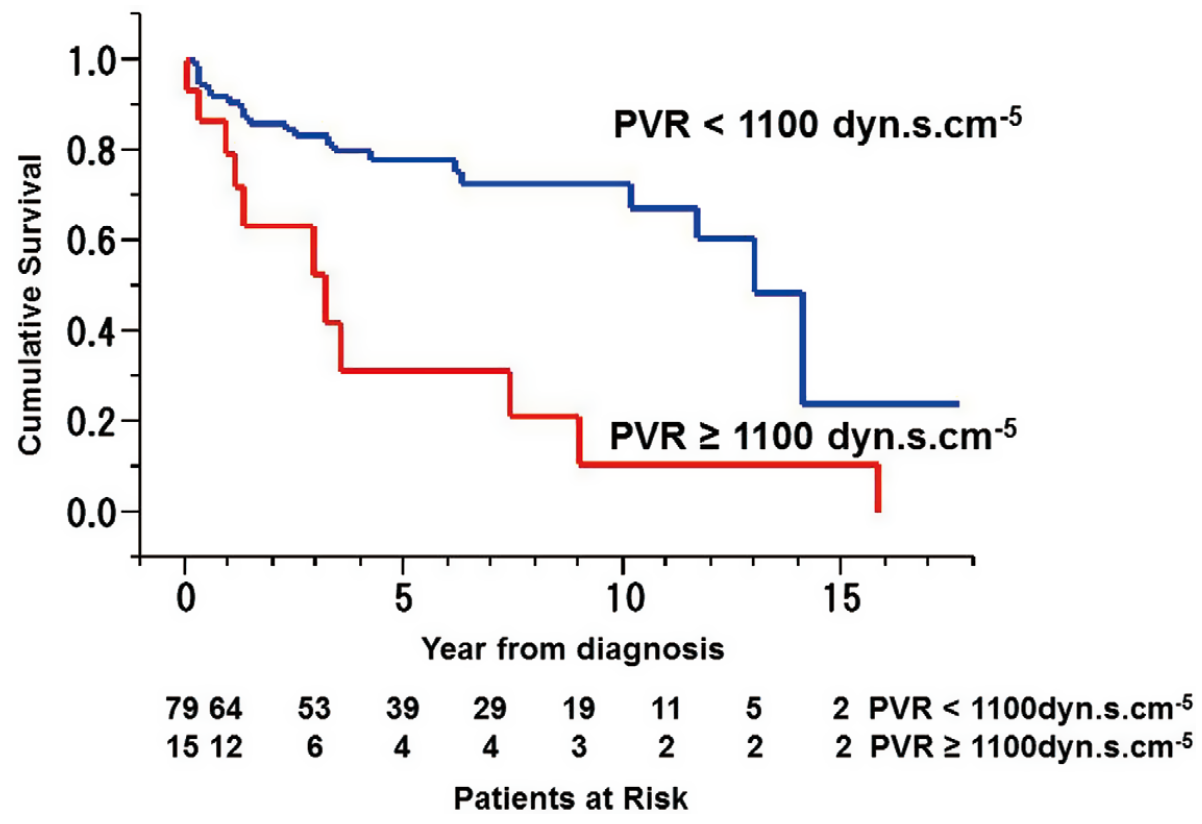

Figure 2. Survival curves according to pulmonary vascular resistance (PVR) $<1,100 \mathrm{dyn} \cdot \mathrm{s} \cdot \mathrm{cm}^{-5}$ and $\geq 1,100 \mathrm{dyn} \cdot \mathrm{s} \cdot \mathrm{cm}-5(\mathrm{n}=95)$. Patients with PVR $\geq 1,100 \mathrm{dyn} \cdot \mathrm{s} \cdot \mathrm{cm}^{-5}$ had a significantly poorer survival than those with PVR $<1,100 \mathrm{dyn} \cdot \mathrm{s} \cdot \mathrm{cm}^{-5}$. Five-year survival in the high PVR group was $31.6 \%$ vs. $78.0 \%$ in the low PVR group ( $P=0.001$; Wilcoxon test).

\begin{tabular}{|c|c|c|c|}
\hline & With $\bmod T x(n=36)$ & Without mod Tx $(n=59)$ & P-value \\
\hline Age (years) & $52.9 \pm 15.2$ & $56.8 \pm 12.6$ & NS \\
\hline Gender (F/M) & $30 / 6$ & $45 / 14$ & NS \\
\hline Duration of symptoms (months) & $32.3 \pm 31.5$ & $27.9 \pm 25.5$ & NS \\
\hline $\mathrm{mPpa}(\mathrm{mmHg})$ & $49.7 \pm 9.81$ & $40.5 \pm 12.2$ & 0.0002 \\
\hline $\mathrm{Cl}\left(\mathrm{L} \cdot \mathrm{min}^{-1} \cdot \mathrm{m}^{-2}\right)$ & $2.38 \pm 0.51$ & $2.72 \pm 0.65$ & 0.008 \\
\hline PVR (dyn $\left.\cdot s \cdot \mathrm{cm}^{-5}\right)$ & $963 \pm 348$ & $727 \pm 447$ & 0.008 \\
\hline $\mathrm{PaO}_{2}$ (torr) & $57.3 \pm 10.1$ & $57.0 \pm 11.3$ & NS \\
\hline WHO functional class $(1 / 2 / 3 / 4)$ & $0 / 6 / 30 / 0$ & $2 / 25 / 25 / 6$ & 0.04 \\
\hline Central disease score $(\leq 1 / \geq 2)$ & $33 / 3$ & $44 / 15$ & 0.04 \\
\hline Comorbidity & 36.1 & 52.5 & NS \\
\hline Coagulation abnormality & 27.8 & 30.5 & NS \\
\hline HLA B5201+ & 29.4 & 46.9 & NS \\
\hline DVT & 33.3 & 35.6 & NS \\
\hline Mod Tx (\%) & 100 & 0 & \\
\hline Bosentan (n) & 10 & 0 & \\
\hline Sildenafil (n) & 17 & 0 & \\
\hline Bosentan+Sildenafil (n) & 6 & 0 & \\
\hline Clinical trial & 3 & 0 & \\
\hline Epoprostenol (n) & 2 & 1 & \\
\hline Beraprost (\%) & 58.3 & 55.9 & NS \\
\hline Oxygen (\%) & 100 & 81.4 & 0.006 \\
\hline Diuretics $(\%)$ & 69.4 & 45.5 & 0.02 \\
\hline Inferior vena cava filter (\%) & 38.9 & 57.6 & NS \\
\hline
\end{tabular}

Data given as mean $\pm \mathrm{SD}, \%$ or $\mathrm{n}$. Abbreviations as in Table 1 .

ing characteristic analysis for death carried out in the present study also identified a similar cut-off of $1,121 \mathrm{dyn} \cdot \mathrm{s} \cdot \mathrm{cm}^{-5}$.

Patients with comorbidities had decreased survival compared with those without these complications (5-year survival rate: $59.3 \%$ vs. $82.9 \%, \mathrm{P}=0.02$ ). Similarly, patients with central disease score $\geq 2$ had a significantly poorer survival rate com- 


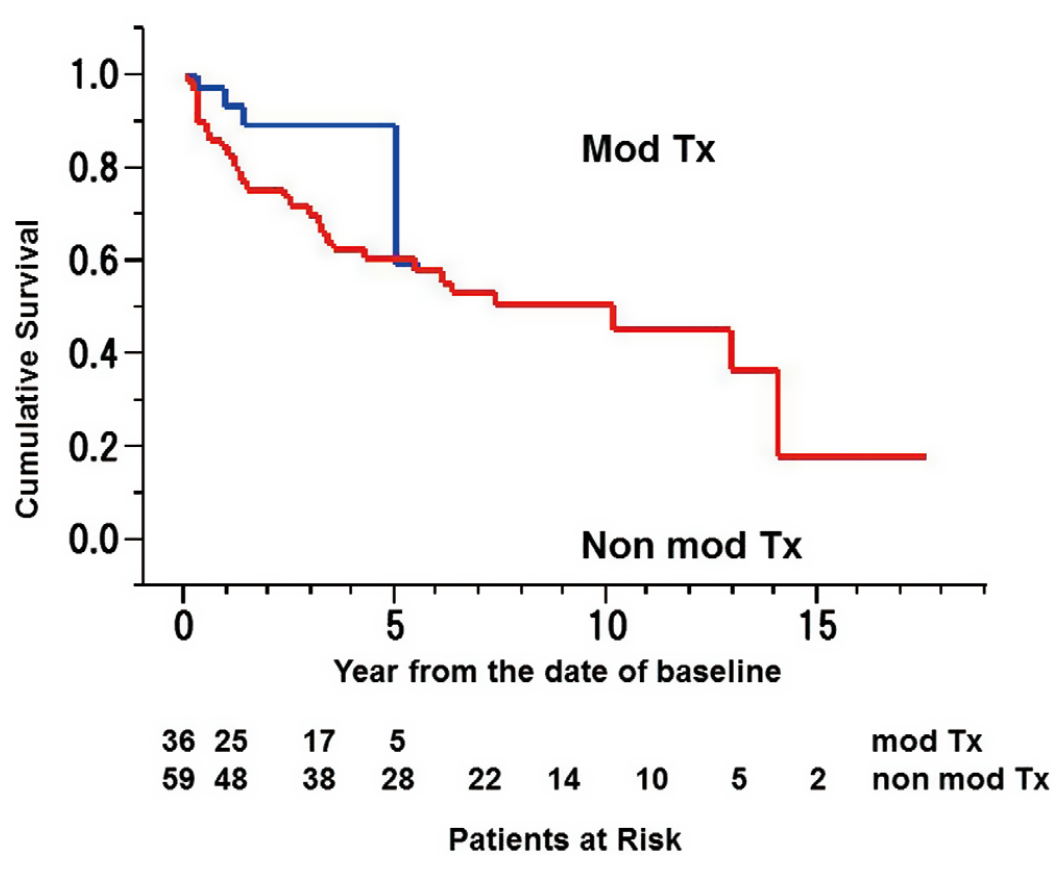

Figure 3. Comparison of survival from the time of initiation of modern oral therapy $(\bmod T x)$ with that from date of diagnosis in patients not taking mod $\mathrm{Tx}$, with the exclusion of mild disease $(n=95)$. Patients not receiving mod Tx had a poorer survival compared with those on mod Tx. Five-year survival was $60.2 \%$ vs. $88.9 \%$ ( $P=0.047$, Wilcoxon test).

pared with those with a score $\leq 1$ (5-year survival rate: $37.5 \%$ vs. $80.0 \%, \mathrm{P}=0.0003$ ). No significant differences were observed in survival curves between patients with and without DVT. There was no significant difference in survival curves between HLA-B*5201-positive and -negative patients (5-year survival rate: $77.7 \%$ vs. $81.4 \%, \mathrm{P}=0.65$ ).

Baseline hemodynamics at diagnosis in the non-mod Tx group and at last catheterization in the mod Tx group were then compared (Table 4). The mPpa and PVR were significantly higher in the mod Tx group compared with the nonmod Tx group $(49.7 \pm 9.81 \mathrm{mmHg}$ vs. $40.5 \pm 12.2 \mathrm{mmHg}$ and $963 \pm 348 \mathrm{dyn} \cdot \mathrm{s} \cdot \mathrm{cm}^{-5}$ vs. $727 \pm 447 \mathrm{dyn} \cdot \mathrm{s} \cdot \mathrm{cm}^{-5}$, respectively). World Health Organization (WHO) functional class was also more severe in the mod Tx group. Pretreatment included beraprost $(n=18)$ and epoprostenol $(n=2)$, while 3 patients were enrolled in the clinical trial. The interval from the date of diagnosis to the initiation of mod-Tx was $2.29 \pm 3.62$ years. Four patients treated with bosentan withdrew prematurely due to adverse events, such as worsening of edema $(n=2)$ or liver dysfunction $(n=2)$. A patient treated with sildenafil withdrew due to severe fatigue $(n=1)$.

The mod Tx group had significantly better survival than the non-mod Tx group (5-year survival: $88.9 \%$ vs. $60.2 \%, \mathrm{P}=0.047$; Figure 3). Univariate Cox proportional hazard analysis showed the use of mod Tx, low PVR (continuous), central disease score $\leq 1$, and lack of comorbidity were associated with better outcome, while multivariate analysis showed that mod Tx was an independent predictor for better outcome, independent of PVR and comorbidities (Table 3).

\section{Discussion}

In 95 medically treated patients after excluding mild disease, patients diagnosed between 2005 and 2010 (group 3) had a significantly better survival rate than patients diagnosed in earlier periods (groups 1 and 2). A higher proportion of group 3 patients were treated with mod Tx. The mod Tx group had better survival compared with the non-mod Tx group, despite their baseline pulmonary hemodynamics being significantly impaired. Multivariate analysis also showed that group 3 or mod Tx were significant predictors for better outcome even after adjustment for PVR, comorbidities, and central disease score. This is the first study to examine survival in medically treated CTEPH patients at follow-up $>20$ years and to show improved survival in patients treated with mod Tx even after adjustment for patient background.

Several issues need to be considered when interpreting these results. First, we identified improved survival in group 3 patients, which included a higher proportion $(65.0 \%)$ of patients treated with mod Tx, compared with the $9.1 \%$ in group 1 and $24.2 \%$ in group 2 . This finding was similar to that of Condliffe et al for non-surgical patients, who found that patients diagnosed from 2003 onwards had significantly better survival and a higher proportion were treated with $\mathrm{PAH}$ therapy (90\%) compared to patients diagnosed between 2001 and 2002 (70\%). ${ }^{8}$ The recent group had been treated more often with mod Tx (ERA, 56\%; PDE-5I, 33\%) compared with the 2001-2002 group (ERA, 17\%; PDE-5I, 0\%). ${ }^{8}$ Condliffe et al did not compare patient background in their 2 groups, although the present study found that group 3 had better survival even after adjustment for patient background, excluding the proportion using pulmonary vasodilators. Multivariate analysis also identified the use of mod Tx as an independent predictor for survival in medically treated patients. It is likely that mod Tx also contributed to improved survival in the recent-treatment Condliffe et al patients.

Second, although there has been no reimbursement of $\mathrm{PAH}$ drugs for CTEPH in Japan or Western countries, this study identified improved outcomes with mod Tx. Riedel et al found that the 5-year survival rate was poor in patients with CTEPH, with approximately $30 \%$ having $\mathrm{mPpa}>40 \mathrm{mmHg} .{ }^{1}$ We agree that CTEPH may be curable by PEA and that this is the standard surgical treatment performed worldwide, mostly at centers of experience. ${ }^{2,3}$ PEA, however, involves considerable 
operative risk, and patients with peripheral type CTEPH, especially those with disproportionally elevated PVR, should be excluded from surgery. ${ }^{2,4}$ Because pulmonary vascular remodeling may be involved in the pathogenesis of CTEPH, similar to that observed in PAH in patients with unobstructed distal pulmonary arteries, medical therapy targeting PAH has been used in inoperable patients or in patients with persistent $\mathrm{PH}$ after surgery.

The first randomized, placebo-controlled trial with bosentan showed significant improvement in pulmonary hemodynamics, although that study failed to show an improvement in 6MWD.7 In another small, randomized, double-blind, placebocontrolled study in 19 CTEPH patients, sildenafil was shown to significantly improve WHO functional class and PVR over 12 weeks, but did not increase 6MWD. The sildenafil-treated group, however, did show an increase in 6MWD after 1 year. ${ }^{14}$

Seyfarth et al reported improved survival in 38 patients treated with specific PAH therapy compared with historical controls. ${ }^{15}$ The present results are similar to theirs, but more specific to ERA and PDE-5I. Further studies from the international registry of CTEPH may provide reliable long-term data, ${ }^{5}$ although randomized clinical trials are necessary to establish recommendations for specific PAH therapy in CTEPH.

Third, the oral prostanoid, beraprost, was approved for PAH medical therapy in Japan in 1999. Ono et al reported a favorable outcome in patients with CTEPH treated with beraprost in a non-randomized trial. ${ }^{16}$ In the current study, we did not find any difference in survival between groups 1 and 2, despite beraprost being used significantly more often in group 2 patients. There was no significant difference in beraprost dose among the 3 groups. The survival in the higher dose group ( $>180 \mu \mathrm{g} /$ day) was not significantly different from that in the lower dose group ( $\mathrm{P}=0.22$; data not shown). In a randomized trial of PAH, Barst et al reported that the beneficial effects of beraprost may occur during the early phase of treatment. ${ }^{17}$ The efficacy of beraprost on long-term survival, however, may be limited in patients with CTEPH.

Fourth, our group previously reported that outcome was improved in medically treated patients with the relatively peripheral type CTEPH (central disease score $\leq 1 ; n=26$ ). ${ }^{18}$ The present study included all the patients from the previous studies and confirmed these findings. Multivariate analysis including the date of diagnosis, showed that relatively peripheral type CTEPH was a significant predictor of better prognosis independent of PVR and comorbidities, whereas on multivariate analysis including the use of mod Tx in patients with PVR $>300 \mathrm{dyn} \cdot \mathrm{s} \cdot \mathrm{cm}^{-5}$, the relatively peripheral type was not significantly associated with survival. In patients with central type CTEPH (central score $\geq 2$ ), mod Tx was used in only 3 of 23 patients, because the majority of patients had died before 2005 , and more recently fewer of such patients were treated medically. The fact that only a few patients with central type CTEPH were treated with mod Tx may have resulted in poor outcome in the central type. This suggests that in patients with the exception of mild disease, the use of mod-Tx may be a more important predictor of outcome than relatively peripheral type CTEPH.

Fifth, we included 3 patients who were treated with epoprostenol. One patient who could not continue bosentan and sildenafil due to side-effects was classified as non-mod Tx, while 2 patients who continued epoprostenol and sildenafil were classified as mod Tx. Survival was calculated from the date of initiation of sildenafil in the mod Tx group. Even after excluding patients treated with epoprostenol, mod Tx was a significant predictor for survival (data not shown) on both univariate and multivariate analysis. The benefit of epoprostenol on survival in CTEPH therefore remains uncertain in this study.

Sixth, Hartz et al reported that perioperative mortality in patients with $\mathrm{PVR} \geq 1,100 \mathrm{dyn} \cdot \mathrm{s} \cdot \mathrm{cm}^{-5}$ was 6 -fold greater than in patients with PVR $<1,100 \mathrm{dyn} \cdot \mathrm{s} \cdot \mathrm{cm}^{-5} \cdot{ }^{13}$ Similarly, Mayer et al reported high operative mortality in patients with PVR $>1,200 \mathrm{dyn} \cdot \mathrm{s} \cdot \mathrm{cm}^{-5} .{ }^{3}$ In this regard, Hoeper et al recommended that patients with advanced small vessel disease with a disproportional elevation in PVR should be treated medically. ${ }^{9}$ The current study found that the survival rate of patients with PVR $<1,100 \mathrm{dyn} \cdot \mathrm{s} \cdot \mathrm{cm}^{-5}$ was considerably better than that of patients with PVR $\geq 1,100 \mathrm{dyn} \cdot \mathrm{s} \cdot \mathrm{cm}^{-5}$. Even in recent medically treated patients, PVR $\geq 1,100 \mathrm{dyn} \cdot \mathrm{s} \cdot \mathrm{cm}^{-5}$ was a significant predictor of poor outcome. Although early recognition of CTEPH and use of PEA without delay in surgical candidates is essential, balloon pulmonary angioplasty (BPA) has recently been performed in Japan, ${ }^{19}$ and it is possible that the combination of BPA and mod Tx may further improve outcome, even in cases of severe PH.

Seventh, the survival rate in patients with comorbidity was significantly poorer than in those without comorbidity, with multivariate analysis showing that comorbidity was a significant predictor of poor outcome. In most cases the comorbidity was either thyroid disease, cancer, or psychiatric disease. Thyroid disease and cancer are considered risk factors for CTEPH, ${ }^{20}$ but no survival analysis including comorbidity has been performed. In Western countries, several studies have shown that AMC is linked to the development of CTEPH and poor survival in both medically and surgically treated CTEPH patients. ${ }^{10,20}$ We were unable to estimate whether AMC was a significant predictor of long-term outcome in Japanese CTEPH patients, because it occurred in only 1 patient who underwent a splenectomy.

Finally, we have previously reported that there was a female predominance in the central type of CTEPH in Japan, which was unrelated to DVT, and that this specific type was associated with HLA-B*5201 positivity. ${ }^{21}$ HLA-B*5201, however, was not a prognostic factor in medically treated patients in the current study.

The main limitation of this study was its retrospective observational design and that data were collected from only one institution. In addition, the indication for mod Tx was physician-oriented and non-randomized. Prospective clinical trials of long-term outcome in patients with CTEPH on mod Tx are therefore warranted.

In conclusion, the survival rate of medically treated CTEPH patients has improved, with the use of mod Tx contributing to better survival.

\section{Acknowledgments}

The present study was supported in part by a grant to the Respiratory Failure Research Group from the Ministry of Health, Labor, and Welfare of Japan, and a research grant from the Ministry of Education, Culture, Sports, Science, and Technology of Japan (22590849).

\section{References}

1. Riedel M, Stanek V, Widimsky J, Prerovsky I. Long-term follow-up of patients with pulmonary thromboembolism: Late prognosis and evolution of hemodynamic and respiratory data. Chest 1982; 81: $151-158$.

2. Galiè N, Hoeper MM, Humbert M, Torbicki A, Vachiery JL, Barbera JA, et al; ESC Committee for Practice Guidelines (CPG). Guidelines for the diagnosis and treatment of pulmonary hypertension: The Task Force for the Diagnosis and Treatment of Pulmonary Hypertension of the European Society of Cardiology (ESC) and the European Respiratory Society (ERS), endorsed by the International Society of Heart and Lung Transplantation (ISHLT). Eur Heart J 2009; 30: 
$2493-2537$.

3. Mayer E, Jenkins D, Linder J, D'Armini A, Kloek J, Meyns B, et al. Surgical management and outcome of patients with chronic thromboembolic pulmonary hypertension: Results from an international prospective registry. J Thorac Cardiovasc Surg 2011; 141: 702-710.

4. Hoeper MM, Mayer E, Simonneau G, Rubin LJ. Chronic thromboembolic pulmonary hypertension. Circulation 2006; 113: 2011-2020.

5. Pepke-Zaba J, Delcroix M, Lang I, Mayer E, Jansa P, Ambroz D, et al. Chronic thromboembolic pulmonary hypertension (CTEPH): Results from an international prospective registry. Circulation 2011; 124: $1973-1981$.

6. Fedullo PF, Auger WR, Kerr KM, Rubin LJ. Chronic thromboembolic pulmonary hypertension. $N$ Engl J Med 2001; 345: 1465-1472.

7. Jaïs X, D'Armini AM, Jansa P, Torbicki A, Delcroix M, Ghofrani HA, et al; for the BENEFiT Study Group. Bosentan for treatment of inoperable chronic thromboembolic pulmonary hypertension. $J \mathrm{Am}$ Coll Cardiol 2008; 52: 2127-2134.

8. Condliffe R, Kiely DG, Gibbs JS, Corris PA, Peacock AJ, Jenkins DP, et al. Improved outcomes in medically and surgically treated chronic thromboembolic pulmonary hypertension. Am J Respir Crit Care Med 2008; 177: 1122-1127.

9. Hoeper MM, Barberà JA, Channick RN, Hassoun PM, Lang IM, Manes A, et al. Diagnosis, assessment, and treatment of non-pulmonary arterial hypertension pulmonary hypertension. J Am Coll Cardiol 2009; 54: S85-S96.

10. Bonderman D, Skoro-Sajer N, Jakowitsch J, Adlbrecht C, Dunkler D, Taghavi $\mathrm{S}$, et al. Predictors of outcome in chronic thromboembolic pulmonary hypertension. Circulation 2007; 115: 2153-2158.

11. JCS Joint Working Group. Guidelines for the diagnosis, treatment and prevention of pulmonary thromboembolism and deep vein thrombosis (JCS 2009): Digest version. Circ J 2011; 75: 1258-1281.

12. Bergin CJ, Sirlin C, Deutsch R, Fedullo P, Hauschildt J, Huynh T, et al. Predictors of patient response to pulmonary thromboendarterectomy. AJR 2000; 174: 509-515.

13. Hartz RS, Byrne JG, Levitsky S, Park J, Rich S. Predictors of mortality in pulmonary thromboendarterectomy. Ann Thorac Surg 1996; 62: $1255-1259$

14. Suntharalingam J, Treacy CM, Doughty NJ, Goldsmith K, Soon E, Toshner MR, et al. Long-term use of sildenafil in inoperable chronic thromboembolic pulmonary hypertension. Chest 2008; 134: 229-236.

15. Seyfarth HJ, Halank M, Wilkens H, Schäfers HJ, Ewert R, Riedel M, et al. Standard PAH therapy improves long term survival in CTEPH patients. Clin Res Cardiol 2010; 99: 553-556.

16. Ono F, Nagaya N, Okumura H, Shimizu Y, Kyotani S, Nakanishi N, et al. Effect of orally active prostacyclin analogue on survival in patients with chronic thromboembolic pulmonary hypertension without major vessel obstruction. Chest 2003; 123: $1583-1588$.

17. Barst RJ, McGoon M, McLaughlin V, Tapson V, Rich S, Rubin L, et al; Beraprost Study Group. Beraprost therapy for pulmonary arterial hypertension. J Am Coll Cardiol 2003; 41: 2119-2125.

18. Yoshimi S, Tanabe N, Masuda M, Sakao S, Uruma T, Shimizu H, et al. Survival and quality of life for patients with distal type chronic thromboembolic pulmonary hypertension. Circ J 2008; 72: 958-965.

19. Sugimura K, Fukumoto Y, Satoh K, Nochioka K, Miura Y, Aoki T, et al. Percutaneous transluminal pulmonary angioplasty markedly improves pulmonary hemodynamics and long-term prognosis in patients with chronic thromboembolic pulmonary hypertension. Circ J 2012; 76: 485-488.

20. Bonderman D, Wilkens H, Wakounig S, Schäfers HJ, Jansa P, Lindner $\mathrm{J}$, et al. Risk factors for chronic thromboembolic pulmonary hypertension. Eur Respir J 2009; 33: 325-331.

21. Tanabe N, Kimura A, Amano S, Okada O, Kasahara Y, Tatsumi K, et al. Association of clinical features with HLA in chronic pulmonary thromboembolism. Eur Respir J 2005; 25: 131-138. 